\title{
ACTUALIZACION DEL REGISTRO DE LEVADURAS CITADAS EN CHILE
}

\section{(Update registry of yeasts cited in Chile)}

\author{
Eduardo Valenzuela ${ }^{1}$ F. \& Oscar $\operatorname{Martínez}^{2}$ V. \\ ${ }^{1}$ Instituto de Microbiología, Facultad de Ciencias, \\ Universidad Austral de Chile. Casilla 167, Valdivia, Chile. \\ ${ }^{2}$ Programa Doctorado en Ciencias de Recursos Naturales, \\ Universidad de La Frontera, Temuco.
}

Palabras claves: Chile, levaduras, taxonomía Key word: Chile, yeasts, taxonomy

\section{RESUMEN}

Se hizo un estudio bibliográfico sobre levaduras citadas para Chile. Con la información recopilada se elaboró un listado actualizado de las levaduras citadas en Chile hasta el año 2007. Las taxa fueron agrupadas de acuerdo a la taxonomía moderna citada por Kirk et al. (2001).

\section{INTRODUCCION}

En la primera mitad del siglo XX, las citas de los hongos reportados en Chile fueron recopiladas por Mújica y Vergara, quienes las publicaron en el libro 'Flora fungosa chilena' en 1945, obra que fue reactualizada y publicada en 1980 por Oehrens (Mújica et al., 1980). De acuerdo a los autores antes señalados, las citas de levaduras solamente se incluyeron en la primera Addenda realizada al texto «Flora fungosa chilena».

En Chile los aportes sobre géneros levaduriformes en el ambiente incluyendo las encontrada en clínica médica (Ferrada, 1946; Honorato, 1948, entre otros), son anteriores a la década del 50 del siglo pasado, por lo que nos basaremos solamente en los posteriores a esta década.

Entre los años 60 al 70 del siglo pasado destacan los aportes de Grinbergs (Grinbergs \& Yarrow, 1970 a,b), quien aisló desde especies arbóreas nativas y suelo varias levaduras nuevas para la ciencia, entre otras pertenecientes a los género Candida ( $\boldsymbol{C}$. chilensis; $\boldsymbol{C}$. valdiviana y $\boldsymbol{C}$.

Recibido el 1 de Noviembre 2007

Aceptado el 17 de Diciembre 2007
ABSTRACT

A bibliographic study on yeasts cited in Chile was carried out. After this, an updated listing of yeasts cited in Chile up to 2007 was made using the gathered information. Taxa were grouped according to the modern taxonomy quoted by Kirk et al. (2001).

tepae) y Rhodotorula (R. auraucariae). A mediados de los 80 del siglo pasado Ramírez y González a partir de maderas en pudrición de distintas especies arbóreas nativas citan nuevas especies de levaduras, pertenecientes a los géneros Candida; Rhodotorula y Schizoblastosporion. Además, estos mismos autores aislan desde díptera una nueva especie, Pichia scaptomyzae. Desde entonces han pasado más de 25 años, período en el cual se han registrado nuevos taxa en varios campos de las ciencias. Esta información se encuentra dispersa, además, la obra de Mujica et al. (1980), no ha sido reactualizada y algunas especies desde el punto de vista taxonómico o de la nomenclatura no corresponden a lo que inicialmente se describió.

Desde la perspectiva taxonómica, las levaduras son un conjunto heterogéneo de hongos, que se encuentran distribuidos en varios géneros pertenecientes a Ascomycota y Basidiomycota (Kirk et al.2001) (Fig.1 y 2).

El objetivo de este trabajo es proporcionar una lista actualizada de levaduras y algunos hongos semejantes a levaduras, citadas en la literatura científica para Chile desde el año 1945 hasta el año 2007, de acuerdo a la taxonomía moderna propuesta por Kirk et al. (2001). 


\section{MATERIALES Y METODOS}

Con el fin de recabar información acerca de las levaduras aisladas en Chile, en primer lugar se recopiló la información citada en Mújica et al. (1980). También se realizó una revisión bibliográfica de revistas científicas del área Micológica y afines (Ej. Boletín Micológico, Revista Chilena Historia Natural, Archivos Medicina Veterinaria, Agricultura Técnica, Revista Médica de Chile, etc.) Además, se revisaron diferentes bases de datos (Ej. CAB, EBSCOhost Web Publishing, The CABI Bioscience and CBS Database of Fungal Names, Scopus, etc.).

Con la información recopilada y teniendo como base la taxonomía de las levaduras que aparece en el Kirk et al. (2001), se elaboraron tablas comparativas de las taxa citadas por Mújica et al. (1980) versus la taxonomía moderna, agregando los nuevos registros para Chile.

\section{RESULTADOS Y DISCUSION}

En el periodo 1980-2007 se han añadido aproximadamente unos 50 taxa a los registros de levaduras citados en la literatura científica para Chile.

En la Tabla 1, primera columna se indican las taxa según Kirk et al. (2001), en la segunda columna las taxa citadas por Mújica et al. (1980). En la última columna se indican las nuevas taxa citadas para Chile entre 1980-2007, acorde a las fuentes bibliográficas consultadas.

En la taxonomía propuesta por Kirk et al. (2001), no se consideran los hongos anamórficos como una unidad taxonómica separada, en cambio, son asignados a su nivel correspondiente en la jerarquía taxonómica de los teleomorfos. En este caso las levaduras anamórficas son distribuidas taxonómicamente entre Ascomycota y Basidiomycota, utilizando la expresión latina Incertae sedis, con la cual se indica que no se tiene total claridad de la ubicación del taxón.

Debe destacarse que este listado, que pretende ser actualizado, posiblemente sólo sea parcial, debido a que no incluye trabajos de autores extranjeros que han recolectado material en Chile y que sus resultados son publicados en revistas científicas internacionales (no consideradas). Además, es posible que el listado de la Tabla 1 no incluya aquellas taxa que son detectadas en trabajos rutinarios, cuyos resultados no son publicados.

Otro aspecto importante a considerar es el cambio de nombre del cual han sido objeto algunas especies de levaduras, como resultado del progreso de la taxonomía. De acuerdo a los datos recopilados, el mayor número de nuevas especies para la ciencia es aportado por Ramírez y González (ver Bibliografía), los que se encuentran detallados en sus publicaciones hechas básicamente en el año 1984. Sin embargo, Ramírez (1988) presentó una rectificación de los nombres de diez especies de levaduras, como se muestra en la Tabla 2. Estas especies que fueron dadas como nuevas especies para la ciencia, después de una exhaustiva revisión, se determinó que no eran especies nuevas, sino sinónimos de otras especies antes descritas. Por otra parte, de acuerdo con CABI Bioscience Database (2007), algunos de los nombres registrados en la Tabla 1 han variado. En la Tabla 3, en la primera columna están las especies cuyos nombres han cambiado, en la segunda columna se registran los nombres actuales de dichas especies y en la tercera columna, se indica la ubicación en la clasificación actual.

\section{AGRADECIMIENTOS}

A la Dirección de Investigación de la Universidad Austral de Chile, y a la beca de la Comisión Nacional de Investigación Científica y Tecnológica, CONICYT, Chile.

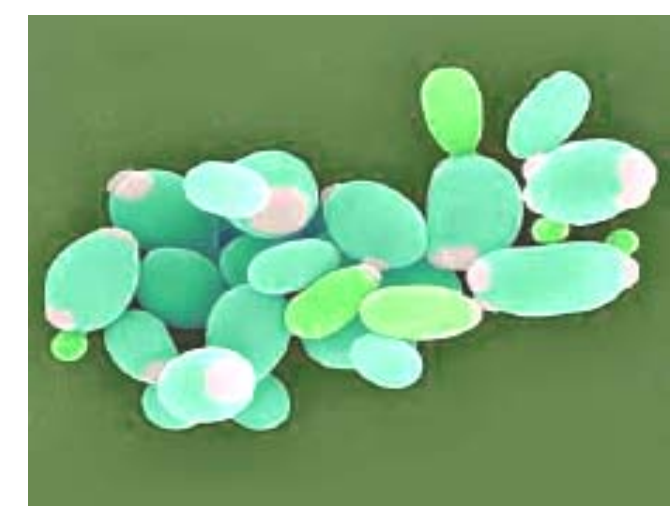

Fig. 2 Candida parapsilosis, tomado de: www.flickr.com/photos/ajc1/1407684424/ 
Tabla 1. Levaduras registradas para Chile.

\begin{tabular}{|c|c|c|}
\hline $\begin{array}{l}\text { Taxa según Kirk et al. } \\
\text { (2001). }\end{array}$ & Citados en Mújica et al. (1980) & $\begin{array}{c}\text { Nuevas Taxa citadas para Chile } \\
\text { (1980-2007) }\end{array}$ \\
\hline \multirow[t]{4}{*}{$\begin{array}{l}\text { Incertae sedis, } \\
\text { Tremellomycetidae, } \\
\text { Basidiomycota }\end{array}$} & & $\begin{array}{l}\text { Apiotrichum eucryphiae C. Ramírez \& } \\
\text { A.E. González } \\
\text { (Citado en Ramírez \& González, } \\
\text { 1984d). }\end{array}$ \\
\hline & & $\begin{array}{l}\text { Apiotrichum futronense C. Ramírez \& } \\
\text { A.E. González } \\
\text { (Citado en Ramírez \& González, } \\
\text { 1984d). }\end{array}$ \\
\hline & & $\begin{array}{l}\text { Apiotrichum nothofagi C. Ramírez \& } \\
\text { A.E. González [as 'Apiotricum'], (Citado } \\
\text { en Ramírez \& González, 1984d). }\end{array}$ \\
\hline & & $\begin{array}{l}\text { Apiotrichum osvaldii C. Ramírez \& } \\
\text { A.E. González } \\
\text { (Citado en Ramírez \& González, } \\
\text { 1984d). }\end{array}$ \\
\hline \multirow[t]{2}{*}{$\begin{array}{l}\text { Saccharomycetaceae, } \\
\text { Ascomycota }\end{array}$} & & $\begin{array}{l}\text { Brettanomyces bruxellensis Kuff. \& } \\
\text { Van Laer, (1921) (Citado en Humeres, } \\
\text { 2006). }\end{array}$ \\
\hline & & $\begin{array}{l}\text { Brettanomyces custersianus Van der } \\
\text { Walt (Citado en Toro \& Piontelli, 1985). }\end{array}$ \\
\hline $\begin{array}{l}\text { Incertae sedis, } \\
\text { Dothideomycetes, } \\
\text { Ascomycetes }\end{array}$ & Blastomyces sp., (Peredo 1960: 8). & \\
\hline \multirow[t]{6}{*}{$\begin{array}{l}\text { Incertae sedis, } \\
\text { Saccharomycetidae, } \\
\text { Ascomycota }\end{array}$} & $\begin{array}{l}\text { Candida albicans (Robin) Berkhout } \\
\text { (Miqueles 1964: 62. Flores 1957:6. Pozo } \\
\text { 1950:13. Alvarez 1962:14.) }\end{array}$ & $\begin{array}{l}\text { Candida ancudensis C. Ramírez \& A.E. } \\
\text { González (Citado en Ramírez \& } \\
\text { González, 1984i). }\end{array}$ \\
\hline & $\begin{array}{l}\text { Candida brumptii Lang. Et Guerra } \\
\text { (Alvarez 1962:14.). }\end{array}$ & $\begin{array}{l}\text { Candida antillancae C. Ramírez \& A.E. } \\
\text { González (Citado en Ramírez \& } \\
\text { González, 1984a). }\end{array}$ \\
\hline & $\begin{array}{l}\text { Candida chilensis Grinbergs et Yarrow } \\
\text { (Grinbergs 1970: 143). }\end{array}$ & $\begin{array}{l}\text { Candida bertae C. Ramírez \& A.E. } \\
\text { González, (Citado en Ramírez \& } \\
\text { González, 1984c). }\end{array}$ \\
\hline & $\begin{array}{l}\text { Candida flareri (Cit. Et Rect.) Lang. Et } \\
\text { Guerra = Torulopsis candida (Saito) } \\
\text { Lodder (Alvarez 1962:14.). }\end{array}$ & $\begin{array}{l}\text { Candida bertae var. chiloensis C. } \\
\text { Ramírez \& A.E. González, (Citado en } \\
\text { Ramírez \& González, 1984c). }\end{array}$ \\
\hline & $\begin{array}{l}\text { Candida guilliermondi (Cast. Lang. Et } \\
\text { Guerra (Miqueles 1964: 62. Honorato } \\
\text { 1948: 106. Pozo 1950:13. Alvarez } \\
\text { 1962:14.). }\end{array}$ & $\begin{array}{l}\text { Candida bondarzewiae C. Ramírez \& } \\
\text { A.E. González (1984) (Citado en } \\
\text { Ramírez \& González, 1984a). }\end{array}$ \\
\hline & $\begin{array}{l}\text { Candida intermedia (Cast. Et And.) } \\
\text { Lang. Et Guerra } \\
\text { (Labatut 1949: 618). }\end{array}$ & $\begin{array}{l}\text { Candida castrensis C. Ramírez \& A.E. } \\
\text { González (1984) (Citado en Ramírez \& } \\
\text { González, 1984a). }\end{array}$ \\
\hline
\end{tabular}




\section{Taxa según Kirk et al. (2001). \\ Citados en Mújica et al. (1980) \\ Candida krusei (Cast.) Basgal \\ (Miqueles 1964: 62. Honorato 1948. A.E González, (Citado en Ramírez \& 108. Aspé 1948:507. Hermosilla 1949: González, 1984f). \\ 621. Pozo 1950: 13. Alvarez 1962: 14). \\ Candida melinii Dissens et Lodder Candida drimydis C. Ramírez \& A.E. (Miqueles 1964: 62.). \\ Candida mycoderma (Reess) Lodder et Candida dubliniensis D.J. Sullivan, Kreger van Rij (Miqueles 1964: 62.). D.C. Coleman (1995) (Citado en Silva et al., 2003c)}

Candida parakrusei (Cast. Et Chalm.) Candida famata (F.C. Harrison) S.A. Lang. Et Guerra = C. parapsilosis (Ash. Mey. \& Yarrow (1978) (Citado en Silva fr.) Lang. Et Talice (Honorato 1948: et al., 2003b)

106. Pozo 1950: 13. Alvarez 1962: 14).

Candida parapsilosis (Ashf.) Lang. Et Candida glabrata (H.W. Anderson) Talice (Miqueles 1964: 62.). (Fig. 2) S.A. Mey. \& Yarrow (1978) (Citado en Tapia et al., 2002).

Candida pelliculosa Red.

Candida huempii C. Ramírez \& A.E. (Pozo 1950: 13). González, (Citado en Ramírez \& González, 1984j).

Candida pseudotropicalis (Cast.) Basgal Candida kefyr (Beij.) Uden \& H.R. (Hermosilla 1949: 779. Pozo 1950: 13). Buckley ex S.A. Mey. \& Ahearn (1983) (Citado en Silva et al., 2003d)

Candida pulcherrima (Lindn.) Candida laureliae C. Ramírez \& A.E. Windisch (Miqueles 1964: 62.). González (Citado en Ramírez \& González, 1984f).

Candida scottii Diddens et Lodder Candida lignophila I. Dill, C. Ramírez (Miqueles 1964: 62.). \& A.E. González (Citado en Dill et al., 1984).

Candida solani Lodder et Kreger van Candida llanquihuensis C. Ramírez \& Rij (Miqueles 1964: 62. Alvarez 1962: A.E. González (Citado en Ramírez \& 14). González, 1984f).

Candida stellatoidea (Jones et Martin) Candida lusitaniae Uden \& Carmo SouLang. Et Guerra (Miqueles 1964: 62.). za (Citado en Toro \& Piontelli, 1985).

Candida tepae Grinbergs

(Grinbergs 1967: 204).
Candida membranifaciens (Lodder \& Kreger) Wick. \& Burton (1954) (Citado en Toro \& Piontelli, 1985).

Candida tropicalis (Cast.) Berkhout Candida mesenterica (A. Geiger) (Peredo 1960: 8. Labatut 1949: 618. Diddens \& Lodder (1942) (Citado en Hermosilla 1949: 774. Honorato 1948: Toro \& Piontelli, 1985).

106. Pozo1950: 13. Alvarez 1962: 14). 


\begin{tabular}{|c|c|c|}
\hline $\begin{array}{l}\text { Taxa según Kirk et al. } \\
\text { (2001). }\end{array}$ & Citados en Mújica et al. (1980) & $\begin{array}{c}\text { Nuevas Taxa citadas para Chile } \\
\text { (1980-2007) }\end{array}$ \\
\hline & $\begin{array}{l}\text { Candida valdiviana Grinbergs et } \\
\text { Yarrow (Grinbergs 1970: 143). }\end{array}$ & $\begin{array}{l}\text { Candida osornensis C. Ramírez \& A.E. } \\
\text { González, (Citado en Ramírez \& } \\
\text { González, 1984f). }\end{array}$ \\
\hline & & $\begin{array}{l}\text { Candida petrohuensis C. Ramírez \& } \\
\text { A.E. González, (Citado en Ramírez \& } \\
\text { González, 1984h). }\end{array}$ \\
\hline & & $\begin{array}{l}\text { Candida pilmaiquenensis C. Ramírez \& } \\
\text { A.E. González, (Citado en Ramírez \& } \\
\text { González, 1984g). }\end{array}$ \\
\hline & & $\begin{array}{l}\text { Candida railenensis C. Ramírez \& A.E. } \\
\text { González (1984) (Citado en Ramírez \& } \\
\text { González, 1984c). }\end{array}$ \\
\hline & $\begin{array}{l}\text { Candida zeylanoides (Cast.) Lang. Et } \\
\text { Guerra (Miquieles 1964: } 62\end{array}$ & $\begin{array}{l}\text { Candida ralunensis C. Ramírez \& A.E. } \\
\text { González (1984) (Citado en Ramírez \& } \\
\text { González, 1984f). }\end{array}$ \\
\hline & Candida sp. (Miquieles 1964: 62). & $\begin{array}{l}\text { Candida rancensis C. Ramírez \& A.E. } \\
\text { González (1984) (Citado en Ramírez \& } \\
\text { González, 1984h). }\end{array}$ \\
\hline & & $\begin{array}{l}\text { Candida rignihuensis C. Ramírez \& } \\
\text { A.E. González, (Citado en Ramírez \& } \\
\text { González, 1984g). }\end{array}$ \\
\hline & & $\begin{array}{l}\text { Candida santjacobensis C. Ramírez \& } \\
\text { A.E. González, (Citado en Ramírez \& } \\
\text { González, 1984i). }\end{array}$ \\
\hline & & $\begin{array}{l}\text { Candida sophiae-reginae C. Ramírez \& } \\
\text { A.E. González, (Citado en Ramírez \& } \\
\text { González, 1984g). }\end{array}$ \\
\hline $\begin{array}{l}\text { Cintractiaceae, } \\
\text { Basidiomycota }\end{array}$ & $\begin{array}{l}\text { Cintractia sorghi (Sorok) de } \mathrm{T} . \\
\text { (Anonimo 1943: } 38 \text { ). }\end{array}$ & \\
\hline \multirow[t]{4}{*}{$\begin{array}{l}\text { Filobasidiaceae, } \\
\text { Basidiomycota }\end{array}$} & $\begin{array}{l}\text { Cryptococcus neoformans (Sanfelice) } \\
\text { Vuillemin (Pozo 1950: 13). }\end{array}$ & $\begin{array}{l}\text { Cryptococcus albidus (Saito) C.E. } \\
\text { Skinner (1950), (Citado en Toro \& } \\
\text { Piontelli, 1985). }\end{array}$ \\
\hline & & $\begin{array}{l}\text { Cryptococcus laurentii (Kuff.) C.E. } \\
\text { Skinner (1950) (Citado en Toro \& } \\
\text { Piontelli, 1985). }\end{array}$ \\
\hline & & $\begin{array}{l}\text { Cryptococcus luteolus (Saito) C.E. } \\
\text { Skinner (1950) (Citado en Toro \& } \\
\text { Piontelli, 1985). }\end{array}$ \\
\hline & & $\begin{array}{l}\text { Cryptococcus uniguttulatus Phaff \& } \\
\text { Fell (Citado en Toro et al. 1993). }\end{array}$ \\
\hline
\end{tabular}




\begin{tabular}{|c|c|c|}
\hline Taxa según Kirk et al. (2001). & Citados en Mújica et al. (1980) & $\begin{array}{c}\text { Nuevas Taxa citadas para Chile } \\
(1980-2007)\end{array}$ \\
\hline \multirow[t]{6}{*}{$\begin{array}{l}\text { Saccharomycetaceae, } \\
\text { Ascomycota }\end{array}$} & $\begin{array}{l}\text { Debaryomyces hansenii } \\
\text { Lodder et Kreger van Rij (Alvarez } \\
\text { 1962: 14). }\end{array}$ & \\
\hline & $\begin{array}{l}\text { Debaryomyces kloeckeri (Gill.) Péju } \\
\text { (Miquelis 1964:62. Alvarez 1962: 14). }\end{array}$ & \\
\hline & $\begin{array}{l}\text { Debaryomyces nicotianae Giovanozzi } \\
\text { (Miquelis 1964: 62). }\end{array}$ & \\
\hline & Debaryomyces subglobosus (Zach.) & \\
\hline & $\begin{array}{l}\text { Lodder et Kreger van Rij(Alvarez } \\
\text { 1962: 14). }\end{array}$ & \\
\hline & $\begin{array}{l}\text { Debaryomyces sp.(Miquelis 1964: } \\
\text { 62). }\end{array}$ & \\
\hline $\begin{array}{l}\text { Saccharomycetaceae, } \\
\text { Ascomycota }\end{array}$ & & $\begin{array}{l}\text { Dekkera intermedia Van der Walt } \\
\text { (Citado en Toro \& Piontelli, 1985; Toro } \\
\text { et al. 1993). }\end{array}$ \\
\hline \multirow[t]{3}{*}{$\begin{array}{l}\text { Incertae sedis, } \\
\text { Ascomycota }\end{array}$} & $\begin{array}{l}\text { Dematium fimbriatum Schw (Bertero } \\
\text { 1829: 611). }\end{array}$ & \\
\hline & $\begin{array}{l}\text { Dematium monophyllum (Schneider } \\
\text { 1887: 686). }\end{array}$ & \\
\hline & $\begin{array}{l}\text { Dematium pullulans D. By (Neger } \\
\text { 1902: 286). }\end{array}$ & \\
\hline \multirow[t]{2}{*}{$\begin{array}{l}\text { Saccharomycopsidaceae, } \\
\text { Ascomycota }\end{array}$} & $\begin{array}{l}\text { Endomycopsis capsularis (Schiön- } \\
\text { nings) Dekk. (Miquelis 1964: 62). }\end{array}$ & \\
\hline & $\begin{array}{l}\text { Endomycopsis fibuliger (Lindn.) } \\
\text { Dekk.(Alvarez 1962: 14). }\end{array}$ & \\
\hline \multirow[t]{3}{*}{$\begin{array}{l}\text { Entylomataceae, } \\
\text { Basidiomycota }\end{array}$} & $\begin{array}{l}\text { Entyloma calendulae (Oud.) D. By. } \\
\text { (Neger 1900: 64. Oehrens 1962: 45). }\end{array}$ & \\
\hline & $\begin{array}{l}\text { Entyloma peullensis } \quad \text { Lindq. } \\
\text { (Lindquist 1960: 193). }\end{array}$ & \\
\hline & Entyloma sp. (Oehrens 1962: 53). & \\
\hline \multirow[t]{3}{*}{$\begin{array}{l}\text { Taphrinaceae, } \\
\text { Ascomycota }\end{array}$} & $\begin{array}{l}\text { Exoascus bullatus (berk. Et Br.) Fckl } \\
\text { (Spegazzini 1918: 101. Spegazzini } \\
\text { 1921: 88). }\end{array}$ & \\
\hline & $\begin{array}{l}\text { Exoascus cerasi Fckl (Graf 1940: } \\
\text { 179). }\end{array}$ & \\
\hline & $\begin{array}{l}\text { Exoascus deformans (berk. Et Br.) } \\
\text { Fckl (Johow 1896: 188. Lavergne } \\
\text { 1900: 519. Lavergne 1902: 91. Reiche } \\
\text { 1909: } 397 .\end{array}$ & \\
\hline
\end{tabular}




\begin{tabular}{|c|c|c|}
\hline Taxa según Kirk et al. (2001). & Citados en Mújica et al. (1980) & $\begin{array}{c}\text { Nuevas Taxa citadas para Chile } \\
(1980-2007)\end{array}$ \\
\hline \multirow[t]{3}{*}{$\begin{array}{l}\text { Exobasidiaceae, } \\
\text { Basidiomycota }\end{array}$} & $\begin{array}{l}\text { Exobasidium antarcticum Speg. } \\
\text { (Spegazzini 1887: 117. Spegazzini } \\
\text { 1887: 276). }\end{array}$ & \\
\hline & $\begin{array}{l}\text { Exobasidium japonicum Shir. } \\
\text { (Oehrens 1963: 44). }\end{array}$ & \\
\hline & $\begin{array}{l}\text { Exobasidium rhododendri Cramer } \\
\text { (Oehrens 1961: 59). }\end{array}$ & \\
\hline
\end{tabular}

Saccharomycetaceae, Ascomycota

Incertae sedis,

Saccharomycetes,

Ascomycota
Hansenula anomala (E.C. Hansen) Syd. \& P. Syd. (1919) (Citado en Toro \& Piontelli, 1985).

\begin{tabular}{|c|c|c|}
\hline & $\begin{array}{l}\text { Geotrichum lactis (= Geotrichum } \\
\text { candidum Lk.) (Álvarez 1962: 14). }\end{array}$ & \\
\hline \multirow{2}{*}{$\begin{array}{l}\text { Incertae sedis, } \\
\text { Saccharomycetes, } \\
\text { Ascomycota }\end{array}$} & $\begin{array}{l}\text { Kloeckera apiculata (Reess emend } \\
\text { Klöcker) Janke (Miqueles 1964: 62). }\end{array}$ & \\
\hline & $\begin{array}{l}\text { Kloeckera corticis (Klöcker) Janke } \\
\text { (Miqueles 1964: 62). }\end{array}$ & \\
\hline \multicolumn{2}{|l|}{$\begin{array}{l}\text { Incertae sedis, } \\
\text { Basidiomycota }\end{array}$} & $\begin{array}{l}\text { Malassezia sp. } \\
\text { (Citado en Rendic et al., 2003). }\end{array}$ \\
\hline \multirow[t]{4}{*}{$\begin{array}{l}\text { Sclerotiniaceae, } \\
\text { Ascomycota, }\end{array}$} & $\begin{array}{l}\text { Monilia albicans (Microtorula) } \\
\text { (Robin) Zopf (= Candida albicans } \\
\text { (Robin) Berkh.) (Prunas 1941:95). }\end{array}$ & \\
\hline & $\begin{array}{l}\text { Monilia cinerea Bon. (Vergara } \\
\text { 1951:88.). }\end{array}$ & \\
\hline & $\begin{array}{l}\text { Monilia laxa (Ehr.) Sacc. Et Vogl } \\
\text { (Morales } 1974: 17 \text { ). }\end{array}$ & \\
\hline & Monilia sp. (Tollenaar 1970 :50). & \\
\hline $\begin{array}{l}\text { Erysiphaceae, } \\
\text { Ascomycota }\end{array}$ & 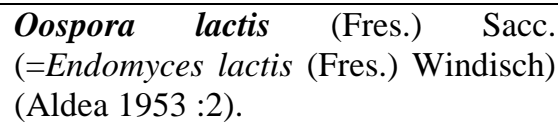 & \\
\hline \multirow[t]{2}{*}{$\begin{array}{l}\text { Saccharomycetaceae, } \\
\text { Ascomycota }\end{array}$} & $\begin{array}{l}\text { Pichia polymorpha Klöcker } \\
\text { (Miqueles 1964: 62) }\end{array}$ & $\begin{array}{l}\text { Pichia guilliermondii Wick. (1966) } \\
\text { (Citado en Toro \& Piontelli, 1985). }\end{array}$ \\
\hline & & $\begin{array}{l}\text { Pichia kudriavzevii Boidin, Pignal \& } \\
\text { Besson (1966) (Citado en Toro \& } \\
\text { Piontelli, 1985). }\end{array}$ \\
\hline
\end{tabular}




Taxa según Kirk et al. (2001). Citados en Mújica et al. (1980)

Nuevas Taxa citadas para Chile (1980-2007)

Pichia scaptomyzae C. Ramírez \& A.E. González (Citado en Ramírez \& González, 1984e).

Pichia vini (Zimm.) Phaff (1956)

(Citado en Toro \& Piontelli, 1985).

Incertae sedis, Urediniomycetes, Basidiomycota
Rhodotorula araucariae Grinbergs et Yarrow (Grinbergs 1970 : 455).

Rhodotorula glutinis (Fres.) Harrison (Miqueles1964 :62.Alvarez 1962: 14).

Rhodotorula minuta (Saito) Harrison (Alvarez $1962: 14$ ).

Rhodotorula mucilaginosa (Jörg.) Harrison (Alvarez 1962 : 14).
Rhodotorula graminis Di Menna (1958)

(Citado en Toro \& Piontelli, 1985).

Rhodotorula grinbergsii (Citado en Ramírez \& González, 1984b).

Rhodotorula nothofagi (Citado en Ramírez \& González, 1985).

(Jörg.) Rhodotorula rubra (Demme) Lodder (Citado en Toro et al. 1993).
Incertae sedis,

Sporidiobolales,

Basidiomycota
Rhodosporidium capitatum Fell, I.L. Hunter \& Tallman (1973) (Citado en Toro \& Piontelli, 1985).

Rhodosporidium infirmominiatum Fell, I.L. Hunter \& Tallman (1973) (Citado en Toro \& Piontelli, 1985).

Saccharomyces fragilis Jörg. (Alvarez $1962: 14)$.

Saccharomycetaceae, Ascomycota
Saccharomyces kluyveri Phaff, M.W. Mill. \& Shifrine (1956) (Citado en Toro \& Piontelli, 1985).

Saccharomyces rosei (Gill.) Lodder et Kreger van Rij (Miqueles $1964: 62$. Alvarez 1962 : 14).

Saccharomyces rouxi Boutroux (Miqueles 1964 :62).

Saccharomyces sp.

(Miqueles 1964:62. Alvarez 1962: 14)

\begin{tabular}{ll}
\hline $\begin{array}{l}\text { Incertae sedis, } \\
\text { Saccharomycetes, } \\
\text { Ascomycota }\end{array}$ & $\begin{array}{l}\text { Schizoblastosporion chiloense C. } \\
\text { Ramírez \& A.E. González [as } \\
\text { 'chiloensis'], (Citado en Ramírez \& } \\
\text { González, 1984j). }\end{array}$ \\
\hline $\begin{array}{l}\text { Incertae sedis, } \\
\text { Ascomycota }\end{array}$ & $\begin{array}{l}\text { Spicaria valdiviensis Speg. } \\
\text { (Spegegazzini } 1910: 181) .\end{array}$ \\
\hline $\begin{array}{l}\text { Incertae sedis, } \\
\begin{array}{l}\text { Urediniomycetes, } \\
\text { Basidiomycota }\end{array}\end{array}$ & $\begin{array}{l}\text { Sporobolomyces holsaticus Windisch } \\
\text { (Alvarez 1962:14). }\end{array}$ \\
\hline
\end{tabular}




\begin{tabular}{|c|c|c|}
\hline Taxa según Kirk et al. (2001). & Citados en Mújica et al. (1980) & $\begin{array}{c}\text { Nuevas Taxa citadas para Chile } \\
(1980-2007)\end{array}$ \\
\hline & $\begin{array}{l}\text { Sporobolomyces sp. } \\
\text { (Alvarez } 1962: 14 \text { ). }\end{array}$ & \\
\hline $\begin{array}{l}\text { Incertae sedis, } \\
\text { Basidiomycetes, } \\
\text { Basidiomycota }\end{array}$ & $\begin{array}{l}\text { Sporotrichum niveum Allesch. Et P. } \\
\text { Henn. (Hennings } 1879: 243 \text { ). } \\
\\
\text { Sporotrichum roseum Pers. } \\
\text { (Bertero } 1828: 195 \text { ). }\end{array}$ & \\
\hline \multirow{13}{*}{$\begin{array}{l}\text { Incertae sedis, } \\
\text { Dothideomycetes, } \\
\text { Ascomycota }\end{array}$} & $\begin{array}{l}\text { Torulopsis aggregata (= Criptococcus } \\
\text { aggregatus) (Flores } 1957: 5 \text { ). }\end{array}$ & \\
\hline & $\begin{array}{l}\text { Torulopsis candida (Saito) Lodder } \\
\text { (Álvarez 1962:14). }\end{array}$ & \\
\hline & $\begin{array}{l}\text { Torulopsis dattila (Kluyver) Lodder } \\
\text { (Miquelis } 1964: 63 \text { ). }\end{array}$ & \\
\hline & $\begin{array}{l}\text { Torulopsis etchellssii Lodder et } \\
\text { Kreger van Rij (Miquelis } 1964: 64 \text { ). }\end{array}$ & \\
\hline & $\begin{array}{l}\text { Torulopsis famata (Harrison) Lodder } \\
\text { et Kreger van Rij (Miquelis 1964 : } 63 . \\
\text { Álvarez 1962:14.). }\end{array}$ & \\
\hline & $\begin{array}{l}\text { Torulopsis fumaginea Speg. } \\
\text { (Spegazzini } 1921: 113 \text { ) }\end{array}$ & \\
\hline & $\begin{array}{l}\text { Torulopsis glabrata (Anderson) } \\
\text { Lodder et de Vries (Miquelis 1964: } \\
\text { 62. Alvarez1962:14 ). }\end{array}$ & \\
\hline & $\begin{array}{l}\text { Torulopsis globosa (Olson et } \\
\text { Hammer) Lodder et Kreger van Rij } \\
\text { (Miquelis 1964:62). }\end{array}$ & \\
\hline & $\begin{array}{l}\text { Torulopsis holmii } \text { (Jörg.) Lodder } \\
\text { (Miquelis 1964: 62). }\end{array}$ & \\
\hline & $\begin{array}{l}\text { Torulopsis inconspicua Lodder et } \\
\text { Kreger van Rij (Alvarez 1962:14. } \\
\text { Miquelis 1964: 62). }\end{array}$ & \\
\hline & $\begin{array}{l}\text { Torulopsis lactis-condensii (Hammer) } \\
\text { Lodder et Kreger van Rij (Alvarez } \\
\text { 1962:14). }\end{array}$ & \\
\hline & $\begin{array}{lrl}\text { Torulopsis } & \text { molischiana } & \text { (Zikes) } \\
\text { Lodder (Miquelis 1964: 62). } & \end{array}$ & \\
\hline & $\begin{array}{l}\text { Torulopsis pinus Lodder et Kreger } \\
\text { van Rij (Miquelis 1964: 62). }\end{array}$ & \\
\hline
\end{tabular}




\begin{tabular}{|c|c|c|}
\hline Taxa según Kirk et al. (2001). & Citados en Mújica et al. (1980) & $\begin{array}{c}\text { Nuevas Taxa citadas para Chile } \\
(1980-2007)\end{array}$ \\
\hline & Torulopsis sp. (Alvarez 1962:14). & \\
\hline \multirow[t]{5}{*}{$\begin{array}{l}\text { Piedraiaceae, } \\
\text { Incertae sedis, } \\
\text { Dothideomycetidae, } \\
\text { Ascomycota, }\end{array}$} & $\begin{array}{l}\text { Trichosporum cutaneum (de Beurm, } \\
\text { Gougerot et Vaucher) Ota (Alvarez } \\
\text { 1962: 14). }\end{array}$ & $\begin{array}{l}\text { Trichosporon asahii Akagi (1929) } \\
\text { (Citado en Silva et al 2003a). }\end{array}$ \\
\hline & $\begin{array}{l}\text { Trichosporum giganteum Unna } \\
\text { (Weinstein } 1953: 32 \text { ). }\end{array}$ & \\
\hline & $\begin{array}{l}\text { Tricosporum margaritiferum (Stautz) } \\
\text { Buchw.(Alvarez 1962: 14). }\end{array}$ & \\
\hline & $\begin{array}{l}\text { Trichosporum pullulans (Lind. Et } \\
\text { Lodder) Diddens (Alvarez 1962: 14). }\end{array}$ & \\
\hline & $\begin{array}{l}\text { Trichosporum sp. (Vaccaro } 1949 \text { : } 82 . \\
\text { Alvarez 1962: 14). }\end{array}$ & \\
\hline
\end{tabular}

Tabla 2. Rectificación de los nombres de las levaduras hechas por Ramírez (1988).

\begin{tabular}{|c|c|}
\hline $\begin{array}{l}\text { Nombre dado por Ramírez y González } \\
\text { en } 1984 .\end{array}$ & $\begin{array}{l}\text { Nombre corregido dado por Ramírez } \\
\text { en 1988. (Sinónimo) }\end{array}$ \\
\hline Apiotrichum eucryphiae C. Ramírez \& A.E. González & $\begin{array}{l}\text { Leucosporidium scottii Fell, Statzell, I.L. Hunter \& } \\
\text { Phaff }\end{array}$ \\
\hline Apiotrichum nothofagi C. Ramírez \& A.E. González & Rhodotorula nothofagi C. Ramírez \& A.E. González \\
\hline Candida bondarzewiae C. Ramírez \& A.E. González & $\begin{array}{l}\text { Candida antillancae sp. nov. C. Ramírez \& A.E. } \\
\text { González }\end{array}$ \\
\hline Candida drymissii C. Ramírez \& A.E. González & $\begin{array}{l}\text { Candida ancudensis sp. nov. C. Ramírez \& A.E. } \\
\text { González }\end{array}$ \\
\hline Candida osornensis C. Ramírez \& A.E. González & Candida parapsilosis (Ashford) Langeron \& Talice \\
\hline Candida pilmaiquenensis C. Ramírez \& A.E. González & Candida maritima (Siepmann) Uden \& H.R. Buckley \\
\hline Candida ralunensis C. Ramírez \& A.E. González & Candida laureliae C. Ramírez \& A.E. González \\
\hline Candida rancensis C. Ramírez \& A.E. González & Metschnikowia pulcherrima Pitt \& M.W. Mill. \\
\hline Candida rignihuensis C. Ramírez \& A.E. González & Candida oleophila Montrocher \\
\hline $\begin{array}{l}\text { Schizoblastosporion chiloensis C. Ramírez \& A.E. } \\
\text { González }\end{array}$ & Candida ingens Van der Walt \& Kerken \\
\hline
\end{tabular}


Tabla 3. Nombres actuales de las levaduras (CABI Bioscience Databases, 2007).

\begin{tabular}{|c|c|c|}
\hline Nombre & Nombre actual & $\begin{array}{c}\text { Clasificación según Kirk } \\
\text { et al. (2001). }\end{array}$ \\
\hline $\begin{array}{l}\text { Candida scottii Diddens \& Lodder } \\
\text { (1942). }\end{array}$ & $\begin{array}{l}\text { Leucosporidium scottii Fell, Statzell, I.L. } \\
\text { Hunter \& Phaff } 1970 .\end{array}$ & $\begin{array}{l}\text { Leucosporidiaceae, } \\
\text { Basidiomycota }\end{array}$ \\
\hline Cintractia sorghi (Sorokin) De Toni. & Endothlaspis sorghi Sorokin 1890. & $\begin{array}{l}\text { Incertae sedis, } \\
\text { Ustilaginales, } \\
\text { Basidiomycota }\end{array}$ \\
\hline $\begin{array}{l}\text { Dematium pullulans de Bary \& } \\
\text { Löwenthal. }\end{array}$ & $\begin{array}{l}\text { Aureobasidium pullulans var. pullulans (de } \\
\text { Bary) G. Arnaud } 1918 .\end{array}$ & $\begin{array}{l}\text { Dothioraceae, } \\
\text { Ascomycota }\end{array}$ \\
\hline $\begin{array}{l}\text { Endomycopsis fibuliger (Lindner) } \\
\text { N.M. Dekker (1931). }\end{array}$ & Endomyces fibuliger Lindner 1907. & $\begin{array}{l}\text { Endomycetaceae, } \\
\text { Ascomycota }\end{array}$ \\
\hline $\begin{array}{l}\text { Exoascus bullatus (berk. Et Br.) } \\
\text { Fckl. }\end{array}$ & $\begin{array}{l}\text { Taphrina bullata (Berk. \& Broome) Tul. } \\
1866 \text {. }\end{array}$ & $\begin{array}{l}\text { Taphrinaceae, } \\
\text { Ascomycota }\end{array}$ \\
\hline Exoascus cerasi Fuckel & Taphrina cerasi (Fuckel) Sadeb. 1890. & $\begin{array}{l}\text { Taphrinaceae, } \\
\text { Ascomycota }\end{array}$ \\
\hline Exoascus deformans (Berk.). & Taphrina deformans (Berk.) Tul. 1866. & $\begin{array}{l}\text { Taphrinaceae, } \\
\text { Ascomycota }\end{array}$ \\
\hline $\begin{array}{l}\text { Hansenula anomala (E.C. Hansen) } \\
\text { Syd. \& P. Syd. (1919). }\end{array}$ & $\begin{array}{l}\text { Pichia anomala (E.C. Hansen) Kurtzman } \\
1984 .\end{array}$ & $\begin{array}{l}\text { Saccharomycetaceae, } \\
\text { Ascomycota }\end{array}$ \\
\hline Monilia cinerea Bonord. (1851). & $\begin{array}{l}\text { Monilinia laxa (Aderh. \& Ruhland) Honey, } \\
\text { in Whetzel } 1945 .\end{array}$ & $\begin{array}{l}\text { Sclerotiniaceae, } \\
\text { Ascomycota }\end{array}$ \\
\hline $\begin{array}{l}\text { Oospora lactis (Fresen.) Sacc. } \\
\text { (1886). }\end{array}$ & Endomyces lactis (Fresen.) Windisch 1951. & $\begin{array}{l}\text { Endomycetaceae, } \\
\text { Ascomycota }\end{array}$ \\
\hline Pichia polymorpha Klöcker (1912). & $\begin{array}{l}\text { Debaryomyces polymorphus (Klöcker) } \\
\text { C.W. Price \& Phaff } 1979 .\end{array}$ & $\begin{array}{l}\text { Saccharomycetaceae, } \\
\text { Ascomycota }\end{array}$ \\
\hline $\begin{array}{l}\text { Rhodosporidium capitatum Fell, I.L. } \\
\text { Hunter \& Tallman (1973). }\end{array}$ & $\begin{array}{l}\text { Cystofilobasidium capitatum (Fell, I.L. } \\
\text { Hunter \& Tallman) Oberw. \& Bandoni, in } \\
\text { Oberwinkler, Bandoni, Blanz \& Kisimova- } \\
\text { Horovitz 1983. }\end{array}$ & $\begin{array}{l}\text { Cystofilobasidiaceae, } \\
\text { Basidiomycota }\end{array}$ \\
\hline $\begin{array}{l}\text { Rhodosporidium infirmominiatum } \\
\text { Fell, I.L. Hunter \& Tallman (1973). }\end{array}$ & $\begin{array}{l}\text { Rhodosporidium diobovatum S.Y. Newell } \\
\text { \& I.L. Hunter, (1970) }\end{array}$ & $\begin{array}{l}\text { Incertae sedis, } \\
\text { Sporidiobolales, } \\
\text { Basidiomycota }\end{array}$ \\
\hline $\begin{array}{l}\text { Saccharomyces fragilis A. Jörg. } \\
\text { (1909). }\end{array}$ & $\begin{array}{l}\text { Kluyveromyces marxianus (E.C. Hansen) } \\
\text { Van der Walt } 1971 .\end{array}$ & $\begin{array}{l}\text { Saccharomycetaceae, } \\
\text { Ascomycota }\end{array}$ \\
\hline $\begin{array}{l}\text { Saccharomyces rouxii Boutroux } \\
\text { (1883). }\end{array}$ & $\begin{array}{l}\text { Zygosaccharomyces rouxii (Boutroux) } \\
\text { Yarrow } 1977 .\end{array}$ & $\begin{array}{l}\text { Saccharomycetaceae, } \\
\text { Ascomycota }\end{array}$ \\
\hline Torulopsis fumaginea (Speg.) Speg. & $\begin{array}{l}\text { Torulopsiella fumaginea (Speg.) Bender } \\
1932 .\end{array}$ & $\begin{array}{l}\text { Incertae sedis, } \\
\text { Ascomycota }\end{array}$ \\
\hline $\begin{array}{l}\text { Torulopsis glabrata (H.W.Anderson) } \\
\text { Lodder \& N.F. de Vries (1939). }\end{array}$ & $\begin{array}{l}\text { Candida glabrata (H.W. Anderson) S.A. } \\
\text { Mey. \& Yarrow, in Yarrow \& Meyer } 1978 .\end{array}$ & $\begin{array}{l}\text { Incertae sedis, } \\
\text { Ascomycota }\end{array}$ \\
\hline $\begin{array}{l}\text { Trichosporum cutaneum (Beurm., } \\
\text { Gougerot \& Vaucher) M. Ota (1926). }\end{array}$ & $\begin{array}{l}\text { Trichosporon cutaneum (Beurm., Gougerot } \\
\text { \& Vaucher) N. Ota } 1926 .\end{array}$ & $\begin{array}{l}\text { Incertae sedis, Tremellales, } \\
\text { Basidiomycota }\end{array}$ \\
\hline $\begin{array}{l}\text { Cryptococcus uniguttulatus } \\
\text { (Wolfram \& Zach) Phaff \& Fell. }\end{array}$ & $\begin{array}{l}\text { Filobasidium uniguttulatum Kwon-Chung } \\
1977 .\end{array}$ & $\begin{array}{l}\text { Filobasidiaceae, } \\
\text { Basidiomycota }\end{array}$ \\
\hline
\end{tabular}




\section{REFERENCIAS}

CABI Bioscience Databases. (2007). The CABI Bioscience and CBS Database of Fungal Names. <http://www.indexfungorum.org/ Names/Names.asp>.

Dill, I.; Ramírez, C. \& González, A. (1984). Candida lignophila sp. nov., a new basidiomycetous yeast anamorph from rotting wood of Drimys winteri.Antonie Van Leeuwenhoek 50:219-25

Ferrada, L. (1946). Contribución al estudio de las levaduras del género Candida. Tesis de Medicina. Univ. de Chile, Santiago.

Grinbergs, J. \& Yarrow, D. (1970a). Two new Candida species:Candida chilensis sp.n. and Candida valdiviana sp.n. Antonie van Leeuwenhoek 36:143-148

Grinbergs, J. \& Yarrow, D. (1970b). Rhodotorula araucariae sp. n. Antonie van Leeuwenhoek 36: 455-457

Honorato, A. (1948). Interpretación del hallazgo de hongos levuriformes (Monilias) en la espectoración. Revista Médica de Valparaiso 1:104-107

Humeres-Vallejos, G.A. (2006). Determinación de levaduras Brettanomyces spp. en vinos chilenos a través de pruebas moleculares. Escuela de Agronomía, Facultad de Ciencias Agronómicas, Universidad de Chile. http://www.cybertesis.cl/tesis/ uchile/2006/humeres_g/html/index-frames.html.

Kirk, P.M.; Cannon, J.C.; David, J.C. \& Stalpers, J.A. (2001). Dictionary of the fungi. 9th Edition CAB Bioscience. CAB International, Cambridge.

Kustzman, C. P. \& Fell, J. W. (1998). The yeast, a taxonomic study. Fourth edition. Elsevier Science B.V. Amsterdam.

Mújica, F. \& Vergara, C. (1945). Flora fungosa chilena. $1^{\text {a }}$ edición. Ministerio de Agricultura, Dirección General Agricultura. Imprenta Stanley, Santiago, Chile. 199 pp.

Mújica, F.; Vergara, C. \& Oehrens, E. (1980). Flora fungosa chilena. $2^{a}$ edición. Revisada y actualizada por Edgar Oehrens B. Universidad de Chile, Facultad de Agronomía. Ciencias Agrícolas $\mathrm{N}^{\mathrm{o}} 5.308 \mathrm{pp}$.

Ramírez, C. (1988). Emendation of yeasts isolated from decayed wood in the evergreen rainy Valdivian Forest of southern Chile. Mycopathologia 103:95-101

Ramírez, C. \& González, A. (1984a). Three new, non-fermenting, and nitrate-negative Candida species isolated the evergreen rainy Valdivian forest of southern Chile. Mycopathologia. 87:175-180

Ramírez, C. \& González, A. (1984b). Rhodotorula grinbergsii sp.nov. isolated from decayed wood in the evergreen rainy Valdivian forest of southern Chile. Mycopathologia 88:51-53

Ramírez, C. \& González, A. (1984c). Two new species and one variety of nitrate-utilizing mycelial Candida isolated from decayed wood in the evergreen rainy Valdivian forest of southern Chile.
Mycopathologia 88:55-60

Ramírez, C. \& González, A. (1984c). Two new species and one variety of nitrate-utilizing mycelial Candida isolated from decayed wood in the evergreen rainy Valdivian forest of southern Chile. Mycopathologia 88:55-60

Ramírez, C. \& González, A. (1984d). Four new species of Apiotrichum isolated from decayed wood in the evergreen rainy Valdivian forest of southern Chile. Mycopathologia 88:71-78

Ramírez, C. \& González, A. (1984e). Pichia scaptomyzae sp. nov., isolated from Scaptomyza multispinosa Malloch (Diptera) in southern Chile. Mycopathologia 88:79-81

Ramírez, C. \& González, A. (1984f). Five new filamentous, glucose-fermenting Candida isolated from decayed wood in the evergreen rainy Valdivian forest of southern Chile. Mycopathologia 88:83-92

Ramírez, C. \& González, A. (1984g). Three new filamentous, fermenting Candida isolated from decayed wood in the evergreen rainy Valdivian forest of southern Chile. Mycopathologia 88:9398

Ramírez, C. \& González, A. (1984h). Two new amycelial Candida isolated from decayed wood in the evergreen rainy Valdivian forest of southern Chile. Mycopathologia 88:99-103

Ramírez, C. \& González, A. (1984i). Candida sant-jacobensis and Candida ancudensis, two new species of yeasts isolated from decayed wood in the evergreen rainy Valdivian forest of southern Chile. Mycopathologia 88:105-109

Ramírez, C. \& González, A. (1984j). Two new filamen-tous, non fermenting Candida and a new Schizoblas-tosporion isolated from decayed wood in the evergreen rainy Valdivian forest of southern Chile. Mycopathologia 88:165-171

Ramírez, C. \& González, A. (1985). Rhodotorula nothofagi sp. nov., isolated from decayed wood in the evergreen rainy Valdivian forest of southern Chile. Mycopathologia. 93:171-173

Rendic, E.; Díaz, C. \& Fich, F. (2003). Presence of the yeast Malassezia in patients with seborrheic dermatitis and subjects without skin lesions. Revista médica de Chile 131:1295-1300

Silva,V.; Zepeda, G. \& Alvarado, D. (2003a). Infección urinaria nosocomial por Trichosporon asahii. Primeros dos casos en Chile. Revista Iberoamericana de Micología 20:21-23

Silva,V.; Zepeda, G.; Rybak, M. \& Febré, N. (2003b). Portación de levaduras en manos de estudiantes de Medicina. Revista Iberoamericana Micología 20:41-45

Silva, V.; Cabrera, M.; Díaz, M.; Abarca, C. \& Hermosilla, G. (2003c). Prevalencia de serotipos de Candida albicans en aislamientos de hemocultivo en Chile y primer caso de candidemia 
por Candida dubliniensis. Revista Iberoamericana Micología 20:46-51

Silva, V.; Díaz, M. \& Febré, N. (2002d). Vigilancia de la resistencia de levaduras a antifúngicos. Revista Chilena de Infectología 19: S 149-156

Tapia, C.; González P.; Díaz, M.C.; Corvalán, V.; Gaete, M.; Cuenca-Estrella, M.; Rodríguez-Tudela, J.L. (2002). Infecciones sistémicas por levaduras en un hospital general. Correlación entre estudio de susceptibilidad in vitro y supervivencia de los pacientes al episodio de infección fúngica. Revista médica de Chile 130:661665
Toro, M.; Díaz, A. \& Piazze, F. (1993). Microhongos y levaduriformes asociados a pimienta negra (Piper nigrum L.). Boletín Micológico 8:77-83

Toro, M. \& Piontelli, E. (1985). Yeasts communities in sandy soil (a beach of V Region, Chile) II. Boletín Micológico 2:109-118 